\section{Milking of Umbilical Cord vs Delayed Cord Clamping}

We read the article by Mangla, et al. [1] which deserves appreciation for the novelty of the topic. We seek clarifications on following issues:

i) In the study outcome, hematocrit values differ by mere $1.8 \%$ and $0.8 \%$ at $48 \pm 6$ hours and at 6 weeks, respectively. This seems to be clinically insignificant, consequently not requiring any interventions.

ii) In babies delivered by caesarean section, anesthesia and surgical interventions interferes with active uterine contraction leading to more blood volume remaining in placenta. Hence, cord will not be refilled after first milking maneuver [2], leading to inefficiency of umbilical cord milking.

iii) At high altitudes [3], infants born to mothers with anemia have higher hematocrits than those born to non-anemic mothers, these factors were not considered while interpreting secondary outcome at 6 weeks of life. Many other studies showed contradictory findings such as no difference on neonatal and maternal outcomes at the age of 48-72 hours in term neonates [4].

We feel that the findings of this study cannot be used to promote umbilical cord milking over delayed cord clamping due to the above noted gaps.

Parvathi Jayaprakash ANd Babu S MAdarkaR* Department of Neonatology, Rainbow Children's Hospital, Bengaluru 560037, Karnataka.

\section{REFERENCES}

*babumadarkar@yahoo.co.in

1. Mangla MK, Thukral A, Sankar MJ, et al. Effect of umbilical cord milking vs delayed cord clamping on venous hematocrit at 48 hours in late preterm and term neonates: A randomized controlled trial. Indian Pediatr. 2020;57:1119-123.

2. Aladangady N, McHugh S, Aitchison TC, et al. Infant's blood volume in a controlled trial of placental transfusion at preterm delivery. Pediatrics. 2006;117:93-8.

3. Ramirez-Cardich ME, Saito M, Gilman RH, et al. Effect of maternal anemia at high altitude on infant hematocrit and oxygenation. Am J Trop Med Hyg. 2004;70:420-4.

4. Panburana $\mathrm{P}$, Odthon T, Pongmee $\mathrm{P}$, et al. The Effect of umbilical cord milking compared with delayed cord clamping in term neonates: A randomized controlled trial. Int $\mathrm{J}$ Womens Health. 2020;12:301-06.

\section{AUTHORS'REPLY}

We thank the readers for their thoughtful comments on our article [1]. Our response is as follows:

i) Our study demonstrated a statistically significant difference of $1.68 \%$ and $1.75 \%$ in the hematocrit values at 48 hours and
6 weeks, respectively, between the two groups. A difference in the hematocrit of $1.75 \%$ - roughly a difference of $6 \mathrm{~g} / \mathrm{L}$ in hemoglobin - at six weeks does indeed look small. However, what constitutes a clinically meaningful difference in a continuous outcome variable is always a matter of debate. One cannot simply disregard the difference in the mean hematocrit without evaluating the risk of other related outcomes like the requirement of transfusion in the first few months of life, failure to thrive, and stunting. Interestingly, a systematic review on daily iron supplementation in children aged 4-23 months showed a mean difference in the hemoglobin values of only $7.2 \mathrm{~g} / \mathrm{L}$ but a considerable reduction in the risk of anemia - by $39 \%$ - in the intervention group [2].

ii) The reference provided for the inefficiency of umbilical cord milking after the first milking maneuver refers to a study that does not include intact-umbilical cord milking but compares early vs. delayed cord clamping (DCC). On the contrary, intact umbilical cord milking has been shown to deliver a more significant placental transfusion than DCC in premature newborns delivered by cesarean section [3].

iii) The comment that infants born to mothers with anemia have higher hematocrits than those born to non-anemic mothers at high altitudes is again not relevant to our study [1] because firstly, the mean hemoglobin (117 vs. $114 \mathrm{~g} / \mathrm{L}$ ) and the proportion of women with anemia were comparable between the two groups, Secondly, the study was conducted in Delhi and not in a high-altitude site; most, if not all, mothers hailed from in and around Delhi. Moreover, the study was a randomized controlled trial, and the randomization process could have taken care of these confounders to a large extent.

Lastly, the study did not conclude that umbilical cord milking should be promoted over of DCC given the significant results; instead, it just stated that the intervention leads to higher venous hematocrit at 48 hours when compared with delayed cord clamping in late preterm and term neonates but the longterm effects of milking need to be further evaluated.

M JeEva SANKAR AND ANU THUKRAL*
Department of Pediatrics.
All India Institute of Medical Sciences,
New Delhi, India.
*dranuthukral@yahoo.com

\section{REFERENCES}

1. Mangla MK, Thukral A, Sankar MJ, et al. Effect of umbilical cord milking vs. delayed cord clamping on venous hematocrit at 48 hours in late preterm and term neonates: A randomized controlled trial. Indian Pediatr. 2020;57:1119-23.

2. Pasricha SR, Hayes E, Kalumba K, et al. Effect of daily iron supplementation on health in children aged 4-23 months: A systematic review and meta-analysis of randomized controlled trials. Lancet Glob Health. 2013;1:e77-86.

3. Katheria AC, Truong G, Cousins L, et al. Umbilical cord milking versus delayed cord clamping in preterm infants. Pediatrics. 2015;136:61-9. 